\title{
An Experimental Study on Percolation Characteristics of a Single-Phase Gas in a Low-Permeability Volcanic Reservoir Under High Pressure
}

\author{
Tang Xiaoyan ${ }^{*}$ \\ College of Geology and Environment, Xi'an University of Science and Technology, Xi'an Shanxi 710054, P.R. China
}

\begin{abstract}
In this paper, we find that with the decrease in the average pore pressure in the process of gas production, both the slippage effect and the stress sensitivity effect will gradually increase; the increase in the slippage effect is significant, while the increase in the stress sensitivity effect is not. In this paper, the Kalamay volcanic gas reservoir of the Junggar Basin in China was selected as the object of our research. The gas reservoir has typical fractured volcanic reservoirs, and the long-term percolation feature remains unclear. To study the percolation characteristics of singlephase gas under high pressure, the experimental method was designed to simulate these characteristics in the process of gas production by measuring the gas flow in the core and the input and the output pressure at both ends. We carried out simulation experiments of single-phase gas flow percolation characteristics under high pressure using 11 pieces of volcanic rock samples in three wells of the study area. The results show that as the core pore pressure increased, the permeability of low-permeability cores of the volcanic rock decreased significantly at room temperature. However, this decrease became more gradual, which means that the higher the core pore pressure is, the smaller the permeability variation caused by gas slippage is; when the pore pressure is above $10 \mathrm{MPa}$, the permeability is nearly constant, slippage effect significantly reduces in the process of gas percolation, so it can be completely ignored under these formation conditions. As the pore pressure decreases, the slippage effect and stress sensitivity effect will gradually increase; when the pore pressure is less than $10 \mathrm{MPa}$, the permeability appears to increase significantly, and this is especially true for a pressure of $5 \mathrm{MPa}$. The main cause of this result is the slippage effect of gas seepage during the depletion of the gas reservoir, when the pore pressure is lower than a certain value. The valid stress changes of the core are not large, and the stress sensitivity is not strong, so the slippage effect plays a major role, which leads to an increase in the gas permeability during the late period of certain flow gas production.
\end{abstract}

Keywords: High pressure, Low permeability, Percolation characteristic, Simulation experiment, Single phase gas, Volcanic rock.

\section{INTRODUCTION}

Volcanic gas reservoirs have become one of the important areas for exploration and development of natural gas in China. Recently, several large-scale volcanic gas reservoirs have been discovered in the Junggar Basin, the Songliao Basin and the Bohai Bay Basin. These reservoirs have reserves of over $3 \times 10^{12} \mathrm{~m}^{3}$ and have become one of controversial issues that have raised concern in the natural gas development of China [1]. The volcanic gas reservoir in the Kalamay gas field of the Junggar Basin is a typical gas reservoir of dual pore medium, whose pore is the main reservoir pore and whose fracture is the primary seepage channel. The gas percolation mechanism in the development of a gas reservoir with a dual pore medium is quite different from that of a homogeneous gas reservoir [2,3]. Because of the complexity of lithology, dissolved pores, dissolved caves and the development of fractures, the percolation mechanism of a dual pore medium reservoir is different from that of a sedimentary sandstone reservoir [4]. As a result, research on the gas percolation characteristics and seepage flow regularity is particularly important in the development of a volcanic gas

*Address correspondence to this author at the College of Geology and Environment, Xián University of Science and Technology, Xián Shanxi, 710054, P.R. China; Tel: 15619362568; E-mail: 1zdtxy2004@sina.com reservoir. To describe the gas seepage regularity correctly and to develop the gas field efficiently, we must determine the gas percolation characteristics [5].

Because of the special pore structure in a lowpermeability gas reservoir $[6,7]$, its percolation characteristics of a single-phase gas is more complex than that of gas reservoirs with moderate permeability and high permeability $[8,9]$. The seepage characteristics of volcanic gas reservoirs, such as the slippage effect, the threshold pressure gradient and the stress sensitivity, had been studied by some scholars [10]; the percolation mechanisms of volcanic reservoirs were also studied. However, all of these experiments were performed under atmospheric pressure conditions [11, 12], which is much different from the actual development conditions of a low-permeability volcanic reservoir. Therefore, it is necessary to perform a study that simulates the percolation characteristics and regularities of gas in a volcanic reservoir under high pressure. In this paper, the research methods used in a low permeability reservoir, i.e., experiments to determine the percolation characteristics of a single-phase gas under high pressure using cores of a natural volcanic gas reservoir, closely approximate the dry-gas reservoir conditions; the research results are more in line with the seepage law of the gas formation condition, which provides the theo- 
retical basis for the efficient development of a volcano rock gas pool.

\section{EXPERIMENTAL PROCESS AND EXPERIMENTAL METHODS}

The cores used in the experiment were selected from Well DIXI 14 and Well DIXI 18 in the Kalamay gas field: one core is from the Well DIXI 14 area, and nine cores are from three wells of the Well DIXI 18 area. The porosity and permeability of the cores after the process of drilling, cutting, grinding and drying were determined (see Table 1). Overall, the core permeability values, which range from $0.007 \mathrm{mD}$ to $0.656 \mathrm{mD}$, essentially cover the absolute permeability distribution of the reservoir in the areas of Well DIXI 14 and Well DIXI 18. Therefore, the flow characteristics of the gas that are studied in the selected cores can reflect the seepage features of the entire area.

The experimental process of determining the gas seepage law of the cores is as follows. First, each of the prepared experimental cores is loaded into the core holder; the injected gas pressure at the core entrance is controlled by the control valve of precise pressure and the outlet pressure is atmospheric pressure; the instantaneous flow rate and cumulative flow are recorded by a gas mass flowmeter. When the gas flow reaches steady state, the inlet pressure of the core is recorded; subsequently, the inlet pressure is changed and the corresponding stable flow is recorded. Generally, the measurement is repeated 20 times, and the corresponding flow is recorded under different pressures. Finally, the corresponding gas permeability of every testing point is calculated using the gas permeability formula and the relationship curves between the permeability and the reciprocal of the average pore pressure are plotted; these curves are analyzed to determine the percolation characteristics and percolation laws of a single-phase gas in low permeability cores of different ranges of permeability.
3. SIMULATING THE HIGH PRESSURE AND STEADY STATE GAS PERCOLATION CHARACTERISTICS WHEN THE OUTLET PRESSURE OF THE CORE REMAINS UNCHANGED

Six low permeability cores were used in experiments: one of them is from Well DX1414, two are from Well DX1824, and three are from Well DX1813. The back pressure of the core outlet, which determined the core entrance pressure, was controlled at $10 \mathrm{MPa}$ during the experiment, and the stable flow of gas in core was measured under the differential pressure of production. Next, the input pressure of core is changed and the corresponding steady flow is measured. Finally, cyclic experiments are performed until the test point meets the required number.

The results indicate that the permeability of cores do not change with the increase of the displacement pressure when the average pore pressure in the volcanic reservoirs cores of low permeability is above $10 \mathrm{MPa}$ see Figs. (1-3). Namely, the permeability decrease caused by the slippage effect was not observed under normal pressure (see Fig. 4).

Here, we compare the permeability versus pressure flow experimental results of three low-permeability cores in Well DX1813 (see Fig. 5 and Fig. 6). The relationship between core permeability and average pore pressure at room temperature is shown in the left curve. The data indicates that the apparent permeability of the core decreases significantly with the increase of the average pore pressure, and the reduction rate of the apparent permeability of the core gradually reduces with the increase of the core pore pressure. In other words, the higher the average pore pressure of the core is, the smaller is the permeability change caused by the gas slippage. When the average pore pressure is above $10 \mathrm{MPa}$, the core permeability is basically constant. The line segment in the right of figure, which is approximately horizontal, provides the relationship between the apparent permeability of the core and the average pore pressure under the back pressure of $10 \mathrm{MPa}$. The apparent permeability is found not to

Table 1. Study of the basic physical parameters of volcanic cores via the gas percolation characteristics.

\begin{tabular}{|c|c|c|c|c|c|c|}
\hline 1 & DX1414 & $5-12 / 42$ & 8.00 & 3.77 & 16.57 & 0.025 \\
\hline 2 & DX1813 & $2-6-12$ & 7.07 & 3.77 & 10.67 & 0.302 \\
\hline 3 & DX1824 & $14-39 / 45$ & 7.06 & 3.76 & 11.4 & 0.116 \\
\hline 5 & DX1813 & $2-2-12$ & 6.85 & 3.76 & 10.9 & 0.656 \\
\hline 6 & DX1813 & $3-6 / 10$ & 7.10 & 3.77 & 11.1 & 0.007 \\
\hline 7 & DX1824 & $16-4 / 45$ & 7.16 & 3.78 & 4.6 & 0.013 \\
\hline 8 & DX1824 & $16-12 / 45$ & 7.83 & 3.78 & 8.6 & 0.025 \\
\hline
\end{tabular}




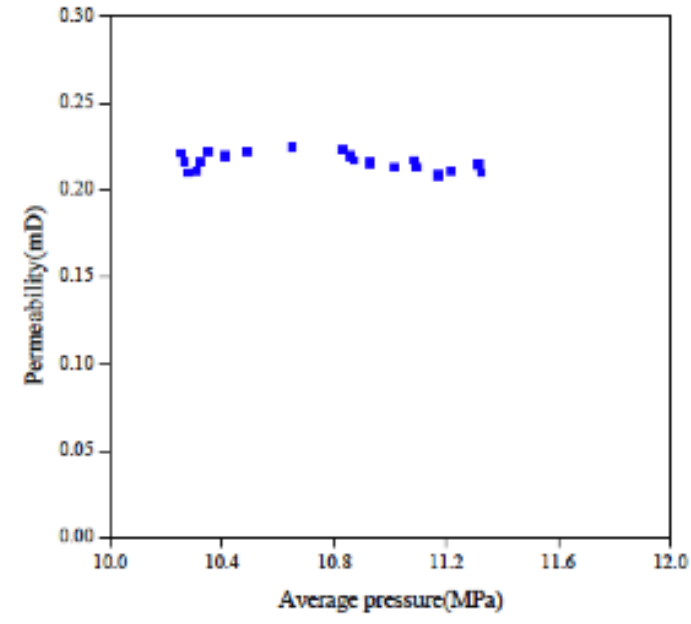

(a) The core No. 2-6/12 of Well DX1813.

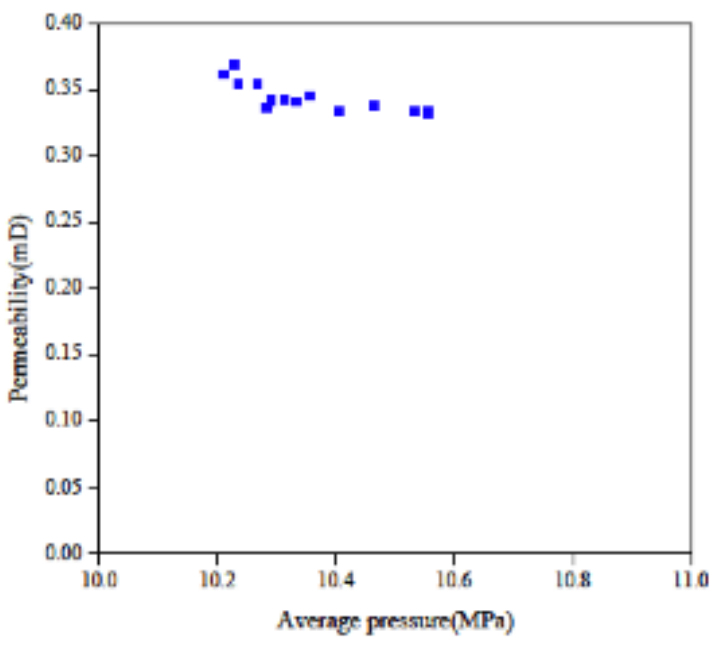

(b) The core No. 2-2/12 of Well DX1813.

Fig. (1). Relationship between the average pore pressure and the apparent permeability of two pieces of the low permeability cores of Well DX1813

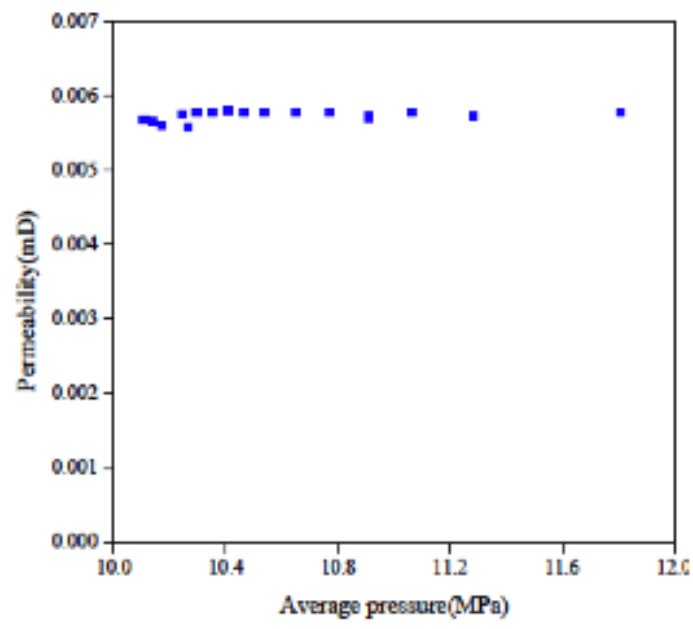

(a) The core No. 3-6/10 of Well DX1813.

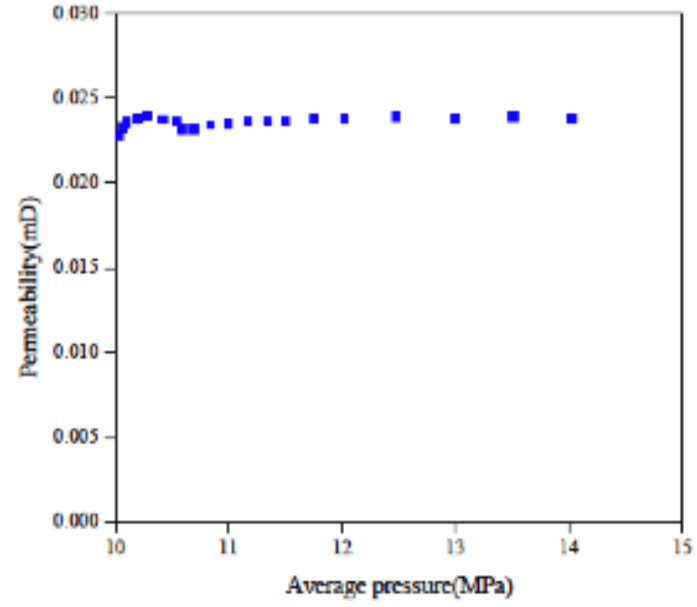

(b) The core No. 5-12/42 of Well DX1414.

Fig. (2). Relationship between the average pore pressure and the apparent permeability of two pieces of the low permeability cores of Well DX1813 and Well DX1414.

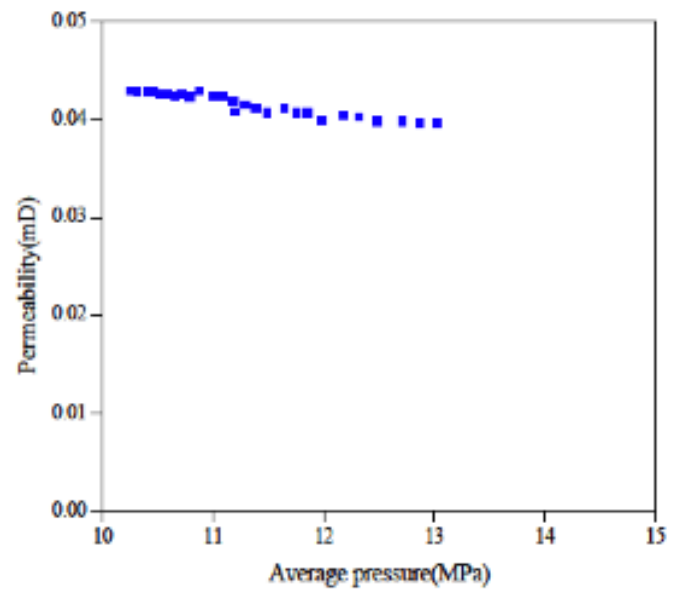

(a) The core No. 14-39/45 of Well DX1824.

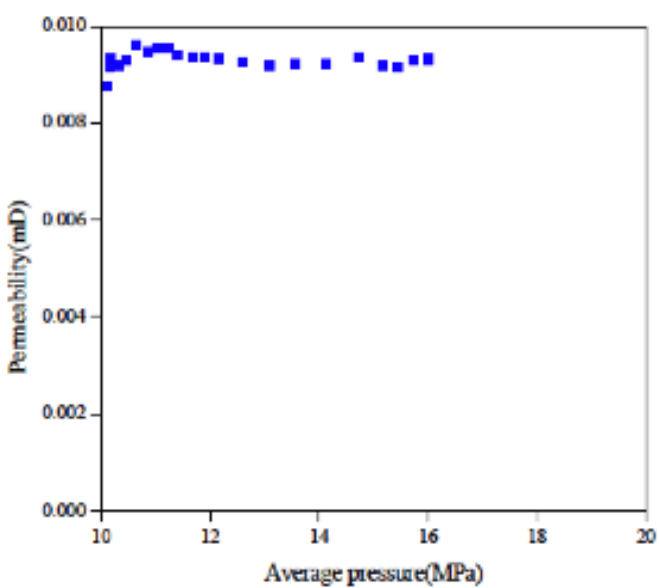

(b) The core No. 15-40/41 of Well DX1824.

Fig. (3). Relationship between the average pore pressure and the apparent permeability of two pieces of low permeability cores of Well DX1824. 


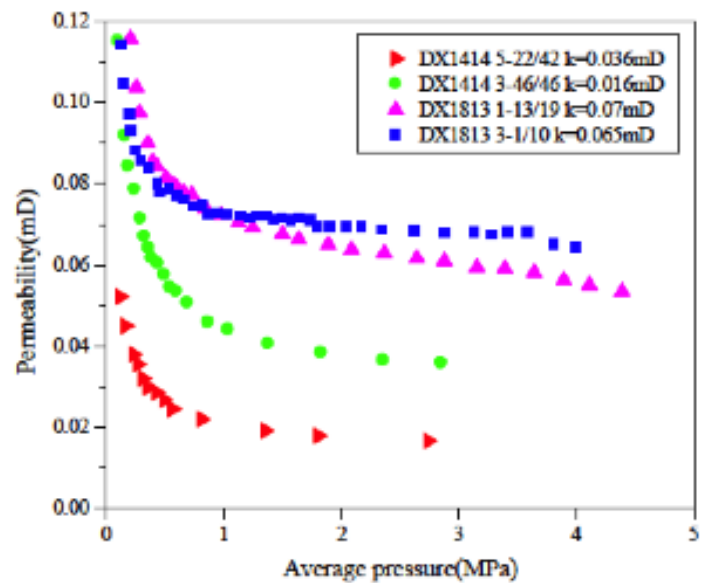

Fig. (4). Relationship between the apparent permeability and the average pore pressure of low permeability cores.

change with the average pore pressure when the back pressure in the core outlet is $10 \mathrm{MPa}$. At this moment, the core permeability tends to be a constant value; that is, when there exists a certain pore pressure (generally greater than $10 \mathrm{MPa}$ ) in a volcanic reservoir of low permeability, the slippage effect during gas percolation significantly decreases and can be completely ignored under the formation conditions. This result is consistent with the one predicted by the gas flow curve of a single phase gas (see Fig. 4). With the increase of the average pore pressure, the apparent permeability of the core gas tested under normal temperature test initially decreases rapidly and then tends to be a constant value, which is the Klinkenberg permeability commonly determined in experiments.

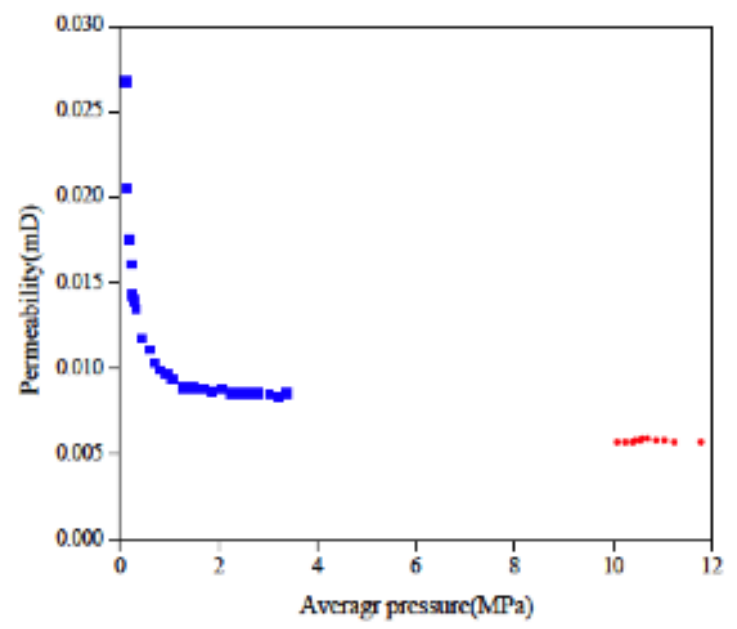

Fig. (6). Relationship between the permeability and the average pressure of core 3-6/10 of Well DX1813 at room temperature and under high pressure.

\section{SIMULATING THE GAS PERCOLATION CHARA- CTERISTICS OF HIGH PRESSURE AND STEADY- STATE WHILE THE OUTLET VELOCITY OF THE CORE REMAINS UNCHANGED}

The cores used in the experiment come from three wells, the cores of Well DX1813 and Well DX1414 are the same as

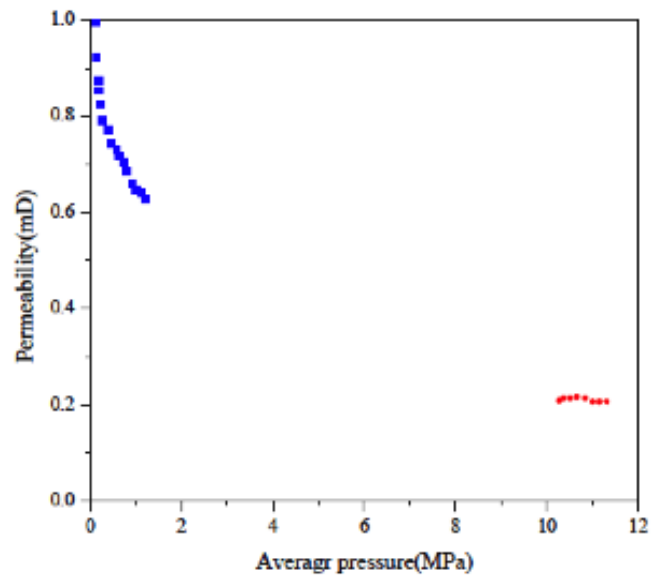

Fig. (5). Relationship between the permeability and the average pressure of core 2-6/12 of Well DX1813 at room temperature and under high pressure.

the cores described above, and the 16-4/45 and 16-12/45 cores are from Well DX1824. During the experiment, we set the confining pressure to $15 \mathrm{MPa}$ greater than the pore pressure and then increased both pressures gradually. Finally, the confining pressure is $50 \mathrm{MPa}$ and the pore pressure is 35 $\mathrm{MPa}$. After the core pressure becomes balanced, we begin an experiment using an ISCO pump, which stably provides the core with constant pressure gas at the core input, and use the export gas mass flow controller to set a constant gas flow of $25 \mathrm{~mL} / \mathrm{min}$. When the distribution of the core pressure is stable, the outlet and the inlet pressures of core are measured under the average pressures of core of approximately 31 $\mathrm{MPa}, 28 \mathrm{MPa}, 24 \mathrm{MPa}, 20 \mathrm{MPa}, 16 \mathrm{MPa}, 12 \mathrm{MPa}, 8 \mathrm{MPa}$, and $5 \mathrm{MPa}$. The experimental data were analyzed, and the experimental results are shown in Figs. (7-9).

Through the experimental results of gas seepage of two cores with developed micro-fracture from Well DX1813 under constant flow velocity of $25 \mathrm{~mL} / \mathrm{min}$ (see Fig. 7), we determine that the change of the gas permeability is not large during the gas production of constant flow. However, when the average pore pressure is lower than $10 \mathrm{MPa}$, core permeability began to obviously increase with the continuing decrease of the pressure. This behavior seems to be contradictory with the observation that the increase of the effective stress results in the decrease of the permeability. Why is this so? After careful analysis, we found that the corresponding pore pressure is less than $10 \mathrm{MPa}$ when the core permeability significantly increases and that the corresponding pore pressure is smaller due to the increase of the permeability. Hence, we conclude that the slippage effect of gas inside the volcanic core was the main cause of this result. That is, the slippage effect and the stress sensitivity effect will gradually increase, which arises from the decrease of the pore pressure in the process of gas production. However, the change in the effective stress in the core body is not large, and the stress sensitivity is not strong; thus, the slippage effect plays a major role, which leads to an increase in the gas permeability in the late period of gas production at a constant rate. The flow curves in Fig. (8) and Fig. (9) both exhibit this trend, which is not as obvious as the trend shown in Fig. (7). The different characteristics of rock fracture and pore structure is the main reason for this difference. The characteristics of the flow 


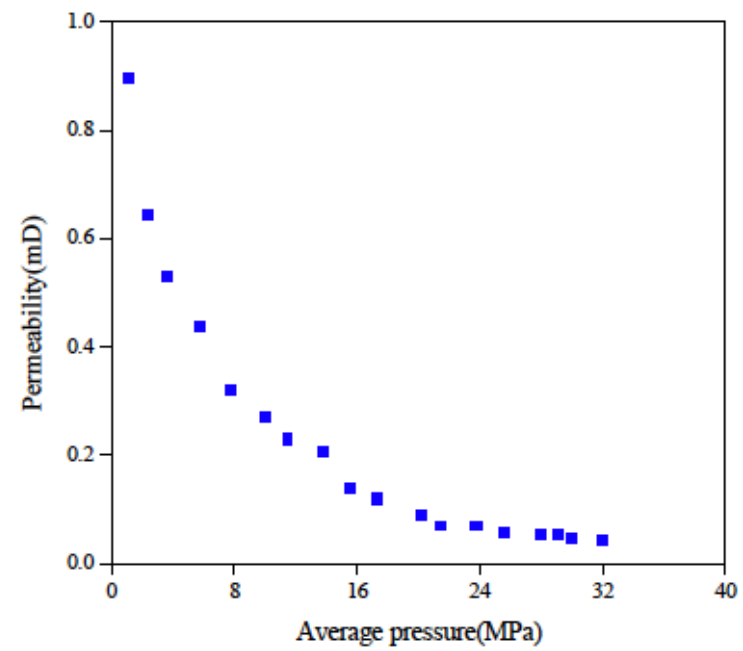

(a) The core No. 2-6/12 of Well DX1813.

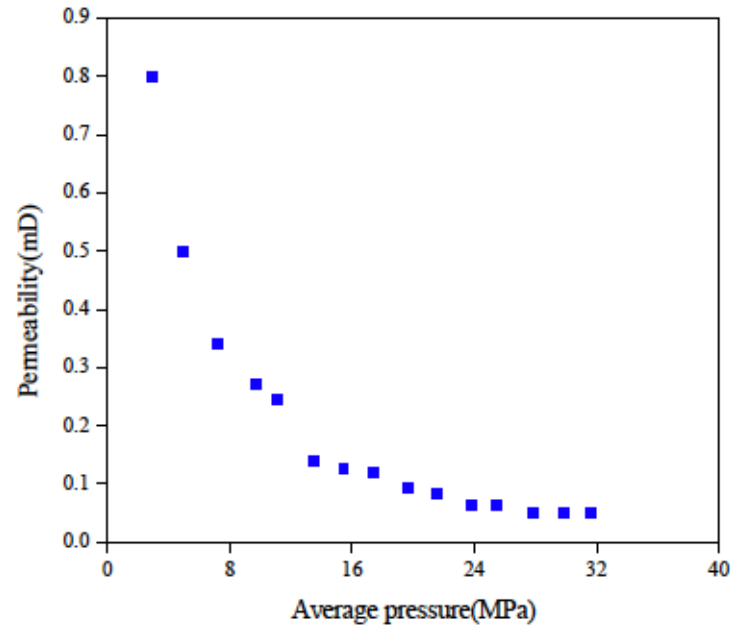

(b) The core No. 2-2/12 of Well DX1813.

Fig. (7). Flow curve of two pieces of low permeability cores of Well DX1813 under high and constant velocity.

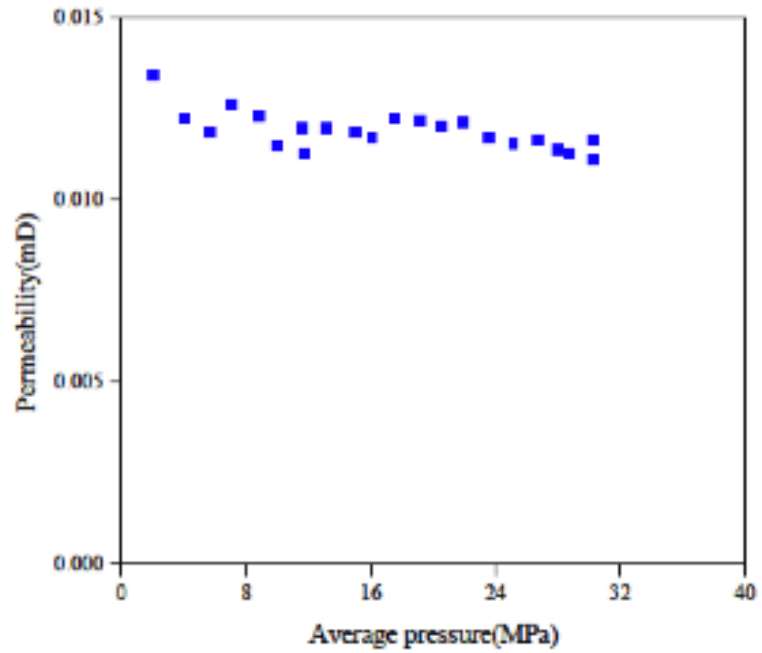

(a) The core No. 3-6/10 of Well DX1813.

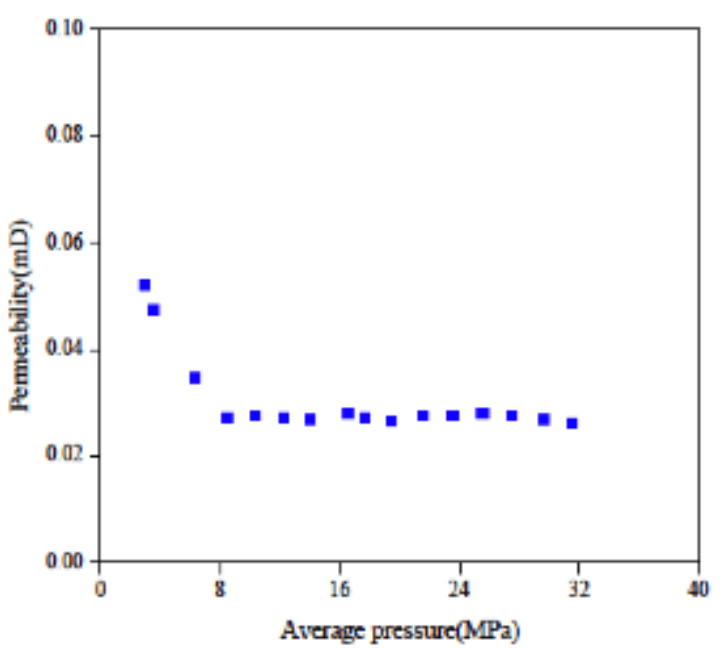

(b) The core No. 5-12/42 of Well DX1414.

Fig. (8). Flow curve of two pieces of extra-low permeability cores of Well DX1813 and Well DX141 under high and constant velocity.

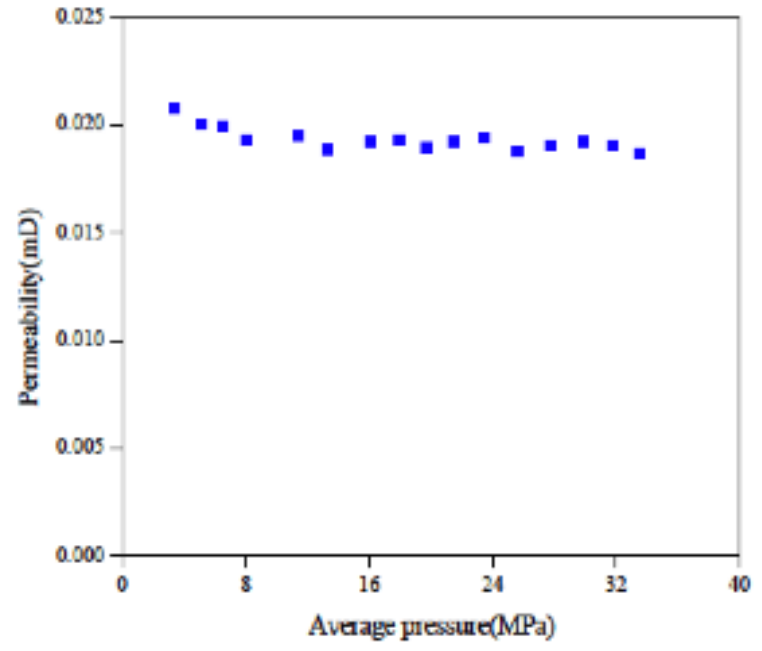

(a) The core No. 16-4/45 of Well DX1824.

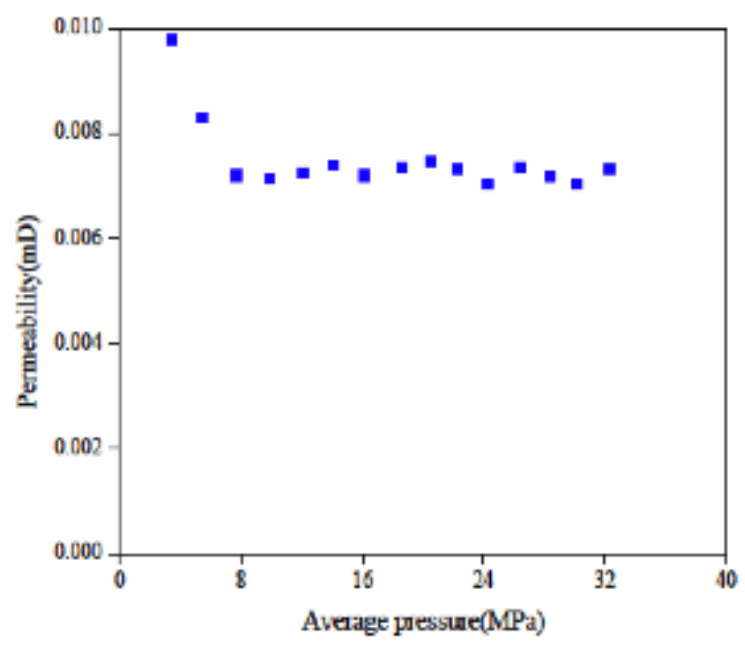

(b) The core No. 12-16/45 of Well DX1824.

Fig. (9). Flow curve of two pieces of extra-low permeability cores under high and constant velocity of Well DX1824. 


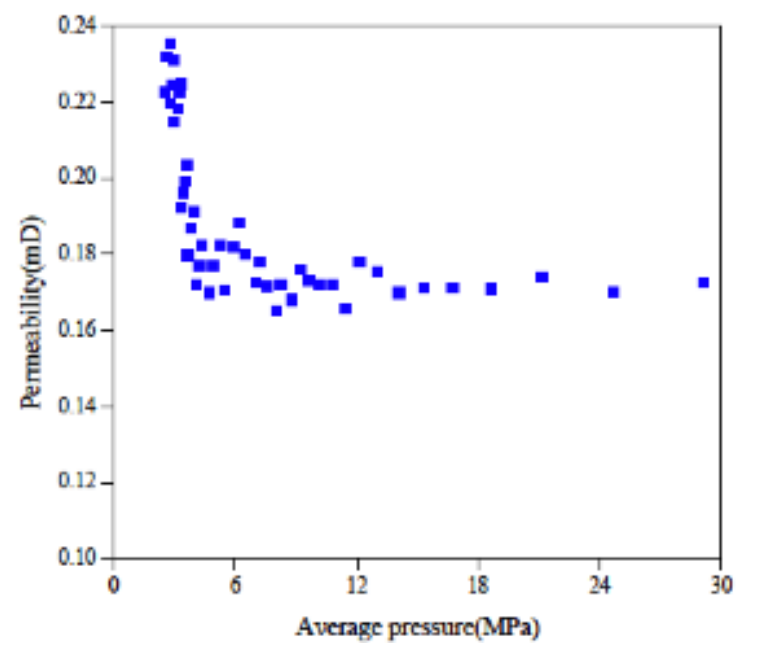

Fig. (10). Flow curve of the low permeability core 14-39/45 of Well DX1824 under high and constant pressure difference.

curve in the three figures indicate that the slippage effect of gas in the late period of gas production is more obvious due to the increase of the core permeability. This finding indicates that the micro-fractures in a volcanic reservoir help to produce the slippage effect. Hence, the more developed are the micro-fractures, the more remarkable is the slippage effect in the late period of development.

\section{SIMULATING THE PSEUDO STEADY-STATE GAS PERCOLATION CHARACTERISTICS DURING THE DEPLETION DEVELOPMENT OF A RESERVOIR}

To simulate the effect of gas production closer to the actual situation, we designed two schemes of the constant pressure difference and the fixed flow, which used two full diameter cores: 16-43/45 and 14-39/45 of Well DX1824.

During the experiment of the percolation characteristics under constant pressure difference and pseudo steady-state, we keep the confining pressure $15 \mathrm{MPa}$ greater than the pore pressure and gradually increase them. The final confining pressure was increased to $50 \mathrm{MPa}$, and the pore pressure was increased to $35 \mathrm{MPa}$. Subsequently, the air inlet was closed for some time until the pressure in the core becomes balanced, and then the experiment is started. To simulate the reservoir conditions that produce the pressure difference, we can set a certain outlet pressure at the core exit by the backpressure valve in the experiment. We tested the input and the output pressures of the corresponding core at different times in the process of depletion development, during which the pore pressure gradually declines from the initial pressure of $30 \mathrm{MPa}$, and eventually, no gas flows out from the output. At this point, the experiment is complete. The experimental data were analyzed, and the experiment results are shown in Fig. (10) and Fig. (11).

It is obvious that during the simulation of the production of low permeable gas, when the average pore pressure of core is above $10 \mathrm{MPa}$, the gas permeability of the core basically is a certain value in the pressure depletion process of production. However, when the average pore pressure is lower than $10 \mathrm{MPa}$, especially at a pressure of $5 \mathrm{MPa}$, the gas permeability of the core significantly increases, which is

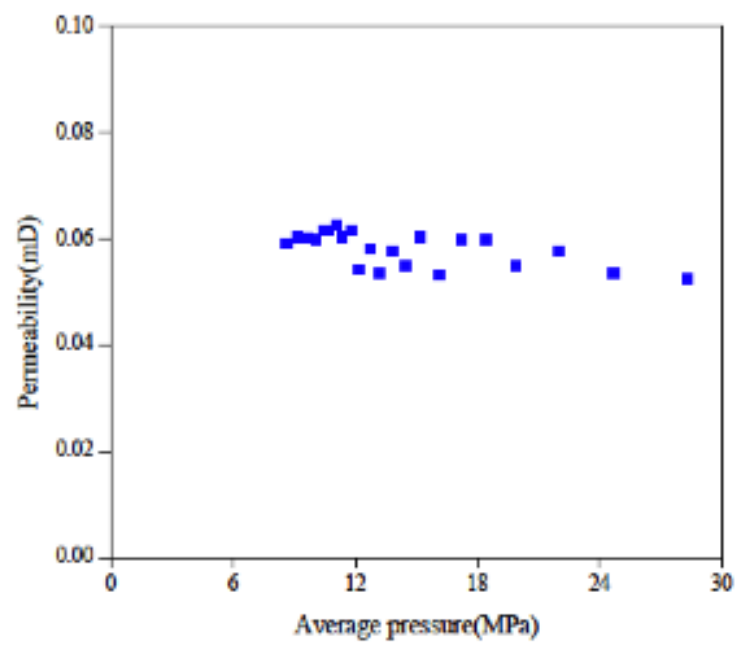

Fig. (11). Flow curve of the extra-low permeability core $16-43 / 45$ of Well DX1824 under high and constant pressure difference.

mainly caused by the slippage effect of gas seepage when the pore pressure is lower than a certain value during depletion production. The results of this study indicate that the stress sensitivity and slippage effects will not generally occur during depletion production of a gas reservoir under the formation conditions.

\section{CONCLUSION}

With the increase of pore pressure, the decrease in the apparent permeability of core gradually reduced; i.e., the higher the average pore pressure of the core is, the smaller is the permeability change caused by gas slippage. When the average pore pressure is above $10 \mathrm{MPa}$, the core permeability is approximately constant. There is a certain pore pressure, which is generally $10 \mathrm{MPa}$ in a volcanic reservoir with low permeability, above which the slippage effect in the process of gas percolation significantly reduces, and it can be completely ignored under this formation condition.

During the gas production of a volcanic reservoir of low permeability, when the average pore pressure of the core is above $10 \mathrm{MPa}$, the gas permeability of the core is a certain value in the pressure depletion process of production. However, when the average pore pressure is lower than $10 \mathrm{MPa}$, especially if it is lower than $5 \mathrm{MPa}$, the gas permeability of the core significantly increases, which is mainly caused by the slippage effect of the gas seepage when the pore pressure is lower than a certain value during depletion production. The results indicate that the stress sensitivity and slippage effects will generally not occur during depletion of a gas reservoir under the formation conditions.

With the decrease of the pore pressure during gas exploitation, the slippage effect and the stress sensitivity will gradually increase. However, the effective stress of the core body does not significantly change, and the stress sensitivity is not strong; thus, the slippage effect plays a major part in the increase of the gas permeability in the late period of constantflow gas production. The higher the core permeability is, the more obvious is the gas slippage effect in the late period of gas production, which indicates that micro-cracks in a volcanic reservoir help to create the slippage effect. We con- 
clude that the more developed are the micro-fractures, the more remarkable is the slippage effect in late development.

\section{CONFLICT OF INTEREST}

The author confirms that this article content has no conflict of interest.

\section{ACKNOWLEDGEMENTS}

Declared none.

\section{REFERENCES}

[1] X. Zhang, S.L. Yang, F.F. Li, H. Chen, X.R. Nie, and J.C. Ding, "Characteristics analysis of gas-water two-phase flow in volcanic gas reservoir", Fault-Block Oil \& Gas Field, vol. 20, no. 5, pp. 631-633, 2013.

[2] M.E. Kennicutt, J.M. Brooks, and R.A. Burke Jr, "Hydrocarbon seepage gas hydrates and antigenic carbonate in the northwestern Gulf of Mexico". In: Presented at Offshore Technology Conference, 1989.

[3] S.A. Khristianovich, "Fundamentals of seepage theory", Journal of Mining Science, vol. 25, pp. 397-412, 1989.

[4] P.F. Fulton, "The effect of gas slippage on relative permeability measurements", Producers Monthly, vol. 15, pp. 14-19, 1951.
[5] M. Hovland, and J. H. Sommerville, "Characteristics of two natural gas seepages in the North Sea", Marine and Petroleum Geology, vol. 2, no. 4, pp. 319-326, 1985 .

[6] Y.Z. Huang, and E.Z. Wang, "Experimental study on coefficient of sensitiveness between percolation rate and effective pressure for low permeability rock", Chinese Journal of Rock Mechanics and Engineering, vol. 26, no. 2, pp. 410-414, 2007.

[7] W.Z. Chen, J.P. Yang, G.W. Wu, X.J. Tan, S.P. Jia, Y.H. Dai, and H.D. Yu, "Experimental study on permeability in low permeability media", Chinese Journal of Rock Mechanics and Engineering, vol. 27, no. 2, pp. 236-243, 2008.

[8] K. Sato, and E. Masahiko, Experimental Study on Gas Seepage in Rock, Groundwater Updates, Germany, Springer, 2000.

[9] W. Xiong, S.S. Gao, Z.M. Hu, H. Xue, and L.Y. Ye, "An experimental study on the percolation characteristics of single phase gas in low and ultra-low permeability sandstone gas reservoirs", Natural Gas Industry, vol. 29, no. 9, pp. 75-77, 2009.

[10] J.X. Dong, M. Tong, B. Ran and N. Li, "Nonlinear percolation mechanisms in different storage-percolation modes in volcanic gas reservoirs", Petroleum Exploration and Development, vol. 40, no. 3, pp. 346-351, 2013.

[11] S.C. Li, X.X. Miao and Z.Q. Chen, "Experimental study on seepage properties of non-Darcy flow in confined broken rocks", Engineering Mechanics, vol. 25, no. 4, pp. 85-92, 2008.

[12] B.S. Ju, and T.L. Fan, "Experimental study and mathematical model of nanoparticle transport in porous media", Powder Technology, vol. 192 , no. 2 , pp. $195-202,2009$.

(C) Tang Xiaoyan; Licensee Bentham Open.

This is an open access article licensed under the terms of the Creative Commons Attribution Non-Commercial License (http://creativecommons.org/licenses/by-nc/4.0/) which permits unrestricted, non-commercial use, distribution and reproduction in any medium, provided the work is properly cited. 